Mots. Les langages du politique

\title{
Le hidjab. Un emprunt autour duquel on glose
}

\section{Geneviève Petiot et Sandrine Reboul-Touré}

\section{OpenEdition}

Journals

Édition électronique

URL : https://journals.openedition.org/mots/781

DOI : 10.4000/mots.781

ISSN : 1960-6001

\section{Éditeur}

ENS Éditions

\section{Édition imprimée}

Date de publication : 1 novembre 2006

Pagination : 49-64

ISBN : 978-2-84788-099-1

ISSN : 0243-6450

\section{Référence électronique}

Geneviève Petiot et Sandrine Reboul-Touré, «Le hidjab. Un emprunt autour duquel on glose », Mots. Les langages du politique [En ligne], 82 | 2006, mis en ligne le 01 novembre 2008, consulté le 23 avril 2022. URL : http://journals.openedition.org/mots/781 ; DOI : https://doi.org/10.4000/mots.781 


\section{Le hidjab. Un emprunt autour duquel on glose}

À la rentrée scolaire de 1989, avec «l'affaire de Creil », lors de polémiques autour du port du voile, en classe, par certaines jeunes filles - voile considéré comme une marque religieuse en contradiction avec la laïcité de l'école -, la presse, pour désigner cet élément vestimentaire, parle de tchador (Petiot, 1995). Quelques années plus tard, nous notons le surgissement du mot hidjab ${ }^{1}$ dans des articles de presse se rapportant, entre autres, aux affrontements en Algérie (état d'urgence en février 1992) et à des évènements plus récents dans l'Hexagone, tels la circulaire Bayrou (octobre 1994) ou la loi du 15 mars 2004 « encadrant, en application du principe de laïcité, le port de signes ou de tenues manifestant une appartenance religieuse dans les écoles, collèges et lycées publics $»^{2}$. L'apparition de ce mot nouveau nous a conduites à analyser des emplois discursifs de hidjab dans la presse écrite française et à explorer l'articulation de l'emprunt, dans un contexte de glose, avec des évènements politiques.

\section{Des gloses : du vêtement au voile}

Nous avons constitué un corpus à partir des bases de données, sur Internet, Lexis Nexis et Factiva et nous avons recueilli environ 400 occurrences de hidjab de 1992 à $2005^{3}$. Ce nombre d'occurrences dans la presse française - et au travers du filtre que constitue une base de données - nous parait relativement faible 4 comparativement à l'impact médiatique et politique du mot. En effet,

1. L'emploi de hidjab devient dominant sans effacer quelques emplois de tchador. Tchador est lexicalisé depuis plusieurs années, par exemple dans le Dictionnaire historique de la langue française, 2000 : "emprunt au persan (sous la forme chadir, 1819, puis tchader, 1852), rare av. le XXe s., répandu en 1978. Le mot désigne le grand voile noir que portent les musulmanes chiites. Diffusé après la révolution islamiste d'Iran (1979), il est devenu synonyme de voile islamique».

2. Voir Annexe 1.

3. L'Agence France Presse, L'Agence Reuters, Le Point, L'Express, Le Monde, Le Figaro, Les Échos, Libération, Le Nouvel Observateur, L'Expansion, Alternatives économiques.

4. En 2006, la recherche sur la base de données Factiva (pour la presse francophone) permet d'obtenir 442 occurrences pour hidjab, 2099 pour foulard islamique et 3216 pour voile islamique.

Université Paris 3, toure@wanadoo.fr 
l'usage de celui-ci a été amplifié par la presse radiophonique et télévisuelle et il a été porté par les débats autour du voile et de la loi du 15 mars 2004. D'un point de vue linguistique, depuis 2005, nous ne pouvons plus parler de néologisme; le mot hidjab est retenu dans le Petit Larousse et dans le Petit Robert avec les définitions suivantes:

Hidjab [idzab] n. m. (mot ar., de hajaba, cacher) Vêtement, en partic. foulard que porte la femme musulmane pour respecter l'obligation de pudeur. (Petit Larousse, 2006)

Hijab ou hidjab ['idzab] n. m. - 1984 - 1989 - mot arabe de hijaba « cacher, voiler » $\checkmark$ Voile qui couvre les cheveux, les oreilles et le cou, porté par de nombreuses musulmanes. (Petit Robert, 2006)

Notons que cet emprunt, présent dans les discours médiatiques et lexicographiques, n'est que faiblement inscrit dans l'usage'.

Le mot hidjab peut être utilisé au fil du discours aussi bien lors des premiers relevés de l'occurrence (1993) que dans les exemples actuels; il n'est donc pas davantage glosé au début de son parcours néologique. Mais cette forme empruntée à l'arabe invite à la formation de gloses :

Quand on travaille sur le vocabulaire d'un corpus, c'est toujours une précieuse trouvaille qu'une séquence métalinguistique autour d'un mot dont la fréquence, particulièrement haute ou basse, a attiré l'attention. Le locuteur arrête le lecteur sur ce mot; le pacte implicite, qui pose le sens comme une évidence partagée, est alors suspendu: le locuteur redéfinit les règles du jeu. (Steuckardt et Niklas-Salminen, 2003, p. 5)

La virgule, qui fait partie des indices, nous permet de relever ci-dessous la glose grande robe noire des wahhabites qui ne laisse voir que les yeux:

Bref, il s'agit de s'intégrer à l'Occident sans en adopter les mœurs, comme en atteste la réislamisation menée par les Frères musulmans via leurs dispensaires ou leurs tontines. Aujourd'hui, hormis dans quelques « vitrines » pour l'étranger, comme les offices du tourisme, les émissions de télévision ou les publicités, où il est totalement proscrit, $90 \%$ des femmes portent le voile, voire le hidjab, la grande robe noire des wahhabites qui ne laisse voir que les yeux. (Les Échos, 5 septembre 2005)

Cette glose nous invite à concevoir le hidjab comme un vêtement et à opposer au niveau sémantique voile et hidjab. Notons que la réalité qui est décrite ici est égyptienne. Or, dans la plupart des gloses du corpus, on ne rencontre pas cette opposition: les journalistes assimilent le hidjab au foulard ou au voile : les gloses apparaissent entre parenthèses à la suite de hidjab, avec ou sans détermination: «(le) foulard», "(le) voile» accompagnent assez régulière-

5. Nous avons interrogé, entre autres, des étudiants en lettres modernes (licence 1, 2 et 3 et Master 1 et 2) à l’Université Paris 3. Le mot reste méconnu. 
ment le xénisme hidjab :

«Mohaïddine, tu n'es pas mort en vain! Mohaïddine, nous te vengerons!» Treize heures, jeudi 2 avril, au cimetière d'El Bireh, petite bourgade palestinienne autonome qui jouxte Ramallah. Huit mille à dix mille Palestiniens, des jeunes en jeans et veste de cuir, des vieillards portant le keffieh traditionnel, quelques centaines de femmes en noir, la chevelure couverte d'un hidjab (foulard), sont venus jusqu'ici accompagner à sa dernière demeure Mohaïddine El Sharif, chef clandestin du Mouvement de la résistance islamique (Hamas), retrouvé mort dimanche 29 mars. (Le Monde, 4 avril 1998)

«Une femme qui porte le hidjab (le voile) dans les circonstances présentes risque d'être agressée par des éléments irresponsables. Donc, elle ne devrait pas le porter », a déclaré Zaki Badawi, directeur du Collège musulman de Londres et président du Conseil des mosquées et des imams. (Agence France Presse, 3 août 2005).

C'est bien le xénisme qui est montré, c'est lui qui constitue le support, le segment glosé. Il n'apparait pas dans le corpus étudié en tant qu'apport, en tant que «segment glosateur» (Steuckardt et Niklas-Salminen, 2005, p.6). Les gloses les plus marquées sont les gloses définitionnelles annoncées, entre autres, par le verbe appeler (elles sont rares dans le corpus étudié) :

Sans revenir sur les nombreux débats au sujet du voile à l'école, il semble nécessaire de préciser ce qu'est justement ce voile.

La notion de pudeur guide l'attitude des femmes et des hommes dans l'islam. Ainsi la tenue ne fait pas partie du culte en lui-même mais est une façon de le pratiquer. Le voile porté par certaines femmes découle de la façon dont elles conçoivent leur pratique de l'islam.

Le vêtement qu'elles portent alors est appelé « hidjab » qui signifie, en arabe, « celui qui empêche». Ainsi est « hidjab» toute chose qui cache. Une femme voilée applique littéralement ce terme, c'est également le cas des hommes ne dévoilant pas leurs jambes.

Par là même, la façon de se couvrir doit se faire selon trois points : le hidjab (qui peut être composé de plusieurs pièces) doit être ample, les différentes pièces qui composent la tenue doivent couvrir l'ensemble du corps à l'exception du visage, des mains et des pieds, enfin le hidjab ne doit pas être transparent. Ensuite chaque femme compose sa tenue, selon l'occasion, la culture du pays où elle vit, son interprétation de l'islam. Certaines se voilent de noir intégral tandis que d'autres portent des hidjab colorés, brodés. (Le Progrès, 30 aout 2004)

Le «marqueur de glose » appelét est particulièrement intéressant pour apprécier le décrochement, le retour auquel il invite sur le mot; on entre alors dans un cadre explicatif, car « gloser, c'est d'abord expliquer: la glose est un acte, son étude part du plan rhétorique pour aller chercher les configurations discursives qui accomplissent cet acte. Plus précisément, gloser, à l'intérieur d'un discours,

6. «Notices », Steuckardt et Niklas-Salminen, 2005, p. 221-227. 
c'est expliquer par un autre la parole lâchée ». (ibid., p. 6)

Le décrochement métalinguistique nous informe sur une traduction possible, "celui qui empêche", et hidjab est présenté en amont comme «le vêtement qu'elles portent alors » et est repris par « toute chose qui cache ». Une autre glose permet au lecteur d'apprendre que le hidjab peut être composé de plusieurs pièces. De sa traduction à sa description, l'emploi du mot hidjab peut ainsi bouleverser la linéarité du dire avec des retours explicatifs.

Au-delà de la glose, le concept de paradigme désignationnel permet de rassembler des mots proposés comme coréférentiels par les journalistes. Pour constituer un paradigme désignationnel, l'analyste puise dans les gloses mais aussi dans un réseau anaphorique entendu au sens large. Nous pouvons alors observer comment le mot hidjab s'insère dans un tissu lexical déjà construit. Pour reprendre l'article du Progrès, riche parce que présentant un contexte explicatif, nous relevons : voile, tenue, vêtement. Ici le vêtement peut être réduit au voile par glissement métonymique.

Les deux reformulations les plus régulières, identifiées par les gloses ou suite à l'élaboration de paradigmes désignationnels, sont voile et foulard. Dans la presse française, le hidjab se réduit donc au voile ou au foulard. Cette particularité se stabilise par la création de deux dénominations, voile islamique et foulard islamique :

En classe économique, les familles ont apporté des draps et des couvertures. Elles les étalent à même le sol, dans les coursives. Cela ne leur fait pas plaisir de se donner en spectacle. Beaucoup se sentent humiliés. «On n’a pas le choix. Si seulement nos sièges étaient inclinables, on pourrait dormir. Mais comment voulez-vous fermer l'œil en restant assis à angle droit ? ", dit un jeune, en montrant sa mère en hidjab (foulard islamique) et sa sœur, déjà allongées par terre, en plein après-midi, pour garder la place. (Le Monde, 11 aout 2005)

Liberté, El Watan et Tribune rapportent aussi que deux dirigeants de l'état-major du GIA, Abderrazak Redjem et Youcef Boubras, ont quitté le mouvement pour protester contre la décision du GIA d'assassiner une fille du président du FIS, Abassi Madani. Ils [les dirigeants du GIA] ont ordonné l'assassinat de la fille d'Abassi Madani parce qu'elle ne porte pas le hidjab (voile islamique). (Agence Reuters, 2 avril 1995)

Dans la presse étudiée, très majoritairement, les journalistes utilisent le mot hidjab qui renvoie à un voile ou un foulard. Ces gloses qui suivent l'emprunt, réduites à un syntagme nominal ou un nom et entre parenthèses, sont régulièrement présentes dans les agences de presse - Reuters et l'Agence France Presse - et on peut se demander s'il n'y aurait pas un effet de «copier-coller» de la part des journalistes qui relaient l'information. Ces gloses n'auraient plus alors de spécificité discursive - choix «militant» du journaliste précisant la valeur de son emprunt - mais elles appartiendraient à la circulation des discours médiatiques. Cependant, quelques gloses sont «situées», notamment par une note de la rédaction: 
Pour lui, les femmes n'étaient pas des êtres humains, elles ne pouvaient pas réfléchir, accuse à la barre Malika Kerri, l'une de ses «épouses », cheveux blonds décolorés et teint blafard. «Il vous a forcée à porter le hidjab [foulard islamique, ndlr] », compatit Joseph Valantin, le président. (Libération, 7 avril 2003)

\section{Un discours polémique}

Dans le corpus retenu, gloser autour du mot permet d'avancer des explications, «gloser» au niveau du texte permet de mettre en place un discours critique, passionné. Nous retiendrons ici quelques exemples qui permettent d'articuler le discours au lexique.

On peut se demander pourquoi les journalistes utilisent le mot hidjab. Il y a sans doute un référent nouveau mais qui peut très bien être dénommé foulard ou voile. Dans la presse, en dehors de quelques emplois dans le domaine de la mode :

Hidjab (voile) rose framboise assorti à son rouge à lèvres Chanel, Ida Royani, quarante-quatre ans, compte à Djakarta parmi les créatrices les plus en vogue de la mode islamique [...] (Le Monde, 2 janvier 1997)

hidjab apparait majoritairement dans des discours polémiques, notamment quand il s'agit de retracer des évènements dans l'Hexagone. Cette polémique est peu marquée dans les gloses mais très présente dans l'ensemble des articles.

Le contexte sociopolitique est un lieu de circulation des débats, à l'oral comme à l'écrit. Il y a bien, dans le cas qui nous intéresse, des partisans pour ou contre le port du voile. L'extrait ci-dessous mentionne différentes associations et il existe même un mouvement dénommé «Pro-hidjab» :

Mis en place, samedi matin, par un conglomérat d'associations britanniques (dont National Assembly against Racism, association musulmane de Grande-Bretagne, et l'Assemblée pour la protection du hidjab, le collectif français « Une école pour toutes et tous » et le collectif des musulmans de France) et chapeauté par le réseau international Prohidjab constitué à la mi-juillet avec le parrainage de Ken Livingstone, le maire de Londres, le séminaire intitulé « Foulard: le droit des femmes de choisir » a tourné à la critique de la loi française sur la laïcité et, dans certaines interventions à la tribune, à la promotion pure et simple du foulard islamique comme « instrument de libération de la femme ». (L'Humanité, 18 octobre 2004)

La polémique a atteint un degré tel que certains journalistes ont créé des néologismes pour dénommer les deux points de vue, pro-hidjab et anti-hidjab :

«Si vous nous laissez vivre selon nos règles - celles qui ne nous sont pas imposées par la société raciste -, vous ferez la preuve de la sincérité de votre antiracisme», explique ainsi Sopo en pastichant les conférences pro-hidjab organisées lors du FSE de Londres, et en soulignant les dangers d'une telle position pour défendre, ensuite, 
l'égalité des hommes et des femmes. (Le Point, 3 novembre 2005)

Animatrice de cantine dans un collège public, douze heures par semaine, elle n'a «pas attendu la loi » pour comprendre qu'elle ne pouvait garder son voile à l'école, même si elle n'est plus collégienne depuis longtemps. "Le jour de l'entretien, la directrice m'a expliqué que le règlement interdisait les symboles religieux. Et je n'ai jamais contesté cette décision.» Puis elle ajoute, avec un air entendu: «Même si je crois que cette loi est anti-hidjab exclusivement. » Dans ce quartier - porte des Postes - à majorité maghrébine, beaucoup pensent comme elle: cette nouvelle législation témoigne à leurs yeux d'« une peur de toute représentation publique de l’islam ». (L'Express, 6 septembre 2004)

Nous voyons ici comment la polémique peut avoir recours à la création lexicale7. Catherine Kerbrat-Orecchioni, dans ses travaux sur la polémique (1980), considère qu'il est nécessaire de distinguer le substantif de l'adjectif : " "une polémique" dénote un échange verbal [...] cependant que, dans les expressions telles que ouvrage, trait, attitude polémique, l'adjectif polémique qualifie la production discursive de l'une seulement des parties en présence, mais dans laquelle nécessairement s'inscrit le discours de l'autre » (Kerbrat-Orecchioni, 1980, p. 9). Ce dernier point est intéressant car, dans les articles de presse, lorsque les journalistes avancent un point de vue, ce dernier se présente en contrepoint d'une idée adverse connue et qui circule dans les esprits des lecteurs mais qui n'est pas forcément retranscrite dans le texte journalistique. II apparait alors une connivence avec le lecteur et cet implicite ne peut prendre place qu'à une époque bien délimitée ; certains articles deviennent difficiles à lire si le contexte politique n'est pas resitué. Ainsi le discours polémique est

un discours disqualifiant, c'est-à-dire qu'il attaque une cible [...], et qu'il met au service de cette visée pragmatique dominante - discréditer l'adversaire, et le discours qu'il est censé tenir - tout l'arsenal de ses procédés rhétoriques et argumentatifs. C'est en particulier un discours saturé [d']axiologiques... (Kerbrat-Orecchioni, 1980, p. 12).

La polémique est portée par les mots et les journalistes utilisent les termes polémique, débat:

Voile islamique. L'affaire des deux sœurs voilées exclues du lycée Henri-Wallon d'Aubervilliers relance la polémique sur le port des insignes religieux. (Sud-Ouest, 25 septembre 2003)

Le gouvernement du président Jacques Chirac propose d'interdire le port du foulard ou voile islamique, le hidjab, dans les établissements scolaires français. D’autres symboles religieux «ostentatoires» doivent également être proscrits, y compris la

7. Nous relevons aussi : «Des mois durant, le débat fit rage entre les pro- et les anti-voiles, bousculant les traditionnels clivages droite gauche... » La Nouvelle République du Centre Ouest, 24 février 2005 . 
kippa ainsi que les "grandes croix chrétiennes». C'est toutefois le hidjab qui se trouve au centre du débat. (Le Monde, 13 janvier 2004)

Les intervenants cherchent à montrer que l'adversaire se trompe :

L'influente confrérie, qui est interdite mais tolérée en Égypte, affirme, dans un communiqué transmis à Reuters, que le chef de l'Etat français s'est mépris sur le rôle du foulard islamique: «La philosophie laïque sur laquelle le président français fonde sa décision [d'interdire les signes religieux ostensibles dans les écoles] en considérant le hidjab comme un symbole religieux n'est pas correcte. Le hidjab islamique est en effet un devoir religieux. » (Agence Reuters, 23 décembre 2003)

Les points de vue s'affrontent et les discours sont « saturés d'axiologiques » :

Les médias arabes, unanimes, condamnent l'«intégrisme laïque» français. (Le Monde, 12 décembre 2003)

Dans ce contexte passionnel ${ }^{8}$, on peut trouver des propos violents. En effet, "l'énoncé polémique intègre et rejette “agressivement”, c'est-à-dire en termes plus ou moins véhéments, voire insultants » (Kerbrat-Orecchioni, 1980, p. 24). Le vote de la loi interdisant le port des signes religieux dans les établissements publics d'enseignement a suscité manifestations et menaces :

Le Groupe salafiste pour la prédication et le combat (GSPC), le plus structuré des groupes islamistes algériens, s'est rapproché, en octobre dernier, du nouveau patron d'al-Qaida en Iran, Abou Moussad al-Zarkaoui, ce même Zarkaoui qui, dans un message sonore du 18 mai dernier, s'en prenait directement à Jacques Chirac, « le chien des croisés, lorsqu'il a ordonné d'ôter le hidjab (voile) des musulmanes en France ». (Le Figaro, 8 juillet 2005)

Notons que les propos violents s'insèrent ici dans du discours rapporté; or, cette forme linguistique participe à la construction de l'argumentation (Doury, 2003 et 2004) :

À lire la presse locale, le nouveau pouvoir des mollahs inquiète les libéraux, et surtout les femmes. Le chroniqueur Khalid Hasan a sonné l'alarme dans le Friday Times de Lahore, mêlant l'ironie et l'horreur dans un éditorial intitulé « Voilà qu'arrive la brigade des barbus»: les femmes sont la principale cible des mollahs, qui «veulent leur faire porter cet horrible objet appelé hidjab. Depuis que le monde existe, elles cherchent de nouveaux moyens de se faire belles, et c'est leur droit. Le hidjab est une invention qui les rend ternes $»$. (Le Monde, 5 décembre 2002)

Rapporter les mots de l'autre permet de ne pas assumer la responsabilité des propos tenus; les propos sont cependant bien dits et même montrés 9 .

8. «Le débat dans l'Hexagone y soulève des passions » (Le Monde, 12 décembre 2003).

9. Les titres des journaux mettent aussi en évidence la controverse, comme on peut le voir dans l'annexe 2. 


\section{Xénisme et modalisation autonymique}

Selon M.-F. Mortureux (2001) :

En règle générale, l'énonciateur qui emprunte un mot étranger le fait parce que, à tort ou à raison, il a le sentiment qu'aucun mot de sa propre langue ne peut désigner le référent dont il veut parler. Mais on peut distinguer deux cas :

- soit le texte présente une réalité étrangère, qui effectivement n’a pas de véritable correspondant en français (oued),

- soit l'emprunt désigne une réalité importée. On peut ainsi opposer tchador et tuner.

Après avoir commenté le deuxième exemple (tuner) qui désigne un objet utilisé en France comme ailleurs, M.-F. Mortureux développe le cas de tchador; on sait que la polémique alors éteinte s'est, depuis, ranimée :

La traduction de tchador par voile masquerait la particularité de la chose, qui est liée à la branche chiite de l'islam, religion d'État en Iran. La polémique qui s'est développée au début de l'année scolaire 1989-1990 concernant le port du voile dans les établissements scolaires publics par des élèves musulmanes s'est apaisée depuis. Lexicalement, elle semble aujourd'hui résolue par l'usage de foulard pour désigner la coiffure des Françaises musulmanes; le tchador reste l'apanage des Iraniennes, désignant de fait un objet différent. Cet emprunt perdure donc en tant que tel, tandis que le mot français foulard, ayant acquis une valeur sémantique particulière, s'est adapté à la réalité nouvelle de la société française. (ibid.)

L'emprunt hidjab succède, dans le temps, à tchador. À l'effroi suscité en Occident par la Révolution islamique iranienne ont succédé les attentats revendiqués par des groupes islamistes, non plus chiites mais sunnites, comme al-Qaïda. Repérer l'emploi d'un emprunt dans un discours relève ainsi du sentiment linguistique des locuteurs. Or, tchador et hidjab sont ressentis comme des emprunts ${ }^{10}$, peu familiers à des yeux ou des oreilles français. Hidjab a ainsi valeur de xénisme, "qui dénomme la notion et connote son origine» (Rey, 1979) : son emploi implique son origine étrangère - ici arabe - et attire l'attention sur elle.

La prononciation du nom pose problème: le français n'a pas de $h$ aspiré, et la transcription peut retenir $d j$ pour noter le $j$ de la racine arabe trilitère à la source du nom : $h-j-b$. Que le $h$ soit ou non réalisé, le nom ne sonne pas français et, pour le moment, aucune réalisation orale ne le rapprocherait de la pho-

10. D'ailleurs, les jeunes filles d'origine maghrébine y reconnaissent un mot de l'arabe classique, distinct du haïk et du safsari de l’arabe maghrébin. 
nologie française ${ }^{11}$. En revanche, l'emploi du déterminant (un, le, son,) le classe parmi les substantifs, ainsi que tchador, et le fait fonctionner comme noyau d'un groupe nominal.

Avec le xénisme, le signe n'est pas transparent et l'altérité se situe à deux niveaux. Au niveau de la forme, plusieurs orthographes en français sont d'ailleurs susceptibles d'être adéquates à la forme orale arabe. C'est pourquoi nous trouvons hijeb, hidjeb, hijab et hidjab ${ }^{12}$, cette dernière forme étant celle retenue pour la diffusion dans la presse écrite. Cette relative instabilité graphique souligne un problème d'adaptation à la morphophonologie du français. «La possibilité du xénisme croît avec l'écart culturel entre la langue d'origine de l'emprunt et la langue réceptrice » (Détrie, Siblot, Vérine, 2001). Le signifiant arrête donc le récepteur. Au niveau sémantique, le xénisme décrit une réalité spécifique inexistante dans l'espace de la langue d'accueil. Cette définition stricte du xénisme est à adapter pour hidjab car, dans l'Hexagone, il y a bien une réalité nouvelle, mais une définition «naturelle» (Martin, 1990) reste encore difficile à formuler ${ }^{13}$. Cette altérité qui crée un arrêt sur le mot nous invite à identifier ici une modalisation autonymique ${ }^{14}$ portée par le signe lui-même :

Forme de dédoublement opacifiant du dire, la modalisation autonymique (désormais MA) présente, structurellement, le cumul d'une référence à la chose et d'une référence au mot par lequel est nommée la chose. L'ordinaire effacement - illusoire - du signe, transparent, "consommé » dans l'accomplissement de sa fonction de médiation est, localement, suspendu : le mot, le moyen du dire, résiste, s'interpose comme corps sur le trajet du dire, et s'y impose comme objet. La MA est une figure de «l'arrêt-sur-mot » (Authier-Revuz, 2003, p. 88-89).

En tant que xénisme, hidjab «s'exhibe » comme mot connoté " arabe » et, de par sa graphie et sa réalisation orale, attire l'attention sur lui. Emprunté à une langue étrangère, il apporte avec son emploi cette étrangeté et, de ce fait, s’il renvoie à un référent comme tous les signes (le mot est alors pris en usage), il invite en même temps à un retour, une relecture - car il peut y avoir une hésitation avant de se familiariser avec le mot en question ; l'usage se double alors d'une mention, le mot est «épinglé » : ce fait relève de la modalisation autonymique. Et, implicitement, on réfère à la religion et à la culture islamiques.

11. Les différentes façons de dire et d'écrire hidjab tiennent aux différentes prononciations arabes et aux oreilles qui les entendent. La transcription des orientalistes pour l'arabe classique est hijâb (avec a long et jim simple); une forme d'insistance apparait avec dj: d'où hidjab. Mais le jim se prononce plutôt $g$ en égyptien, d'où higâb, et se prononce dj au Maghreb, d'où l'algérien hidjeb (où le â long devient plus nettement $e$ ouvert). À comparer avec jabal (« la montagne », en arabe classique), qui donne gabal (en égyptien) et djebel (en algérien). Nous remercions chaleureusement Maurice Tournier pour ces précisions.

12. Le Petit Robert 2006 propose deux orthographes : hijab et hidjab.

13. Voir note 5.

14. Par ailleurs, la modalisation autonymique est conviée par différentes formes (la virgule, les parenthèses, le verbe appeler...) qui sont plus « classiques » avec les gloses comme ci-dessus. 


\section{Une nouvelle organisation lexico-sémantique}

L'intitulé de la loi du 15 mars 2004 comporte donc une composition originale - signes ou tenues manifestant une appartenance religieuse - qui fonctionne comme un hyperonyme $\mathrm{e}^{15}$ discursif et juridique. Cette création lexicale réorganise sémantiquement certaines coiffures et certains signes religieux. Le voile et autres reformulations désignant les modes de coiffure (voire de vêtements) de musulmanes revendiquant les tenues traditionnelles ne sont plus qu'une partie de cet hyperonyme : kippa (juive), croix (chrétienne), turban (sikh), etc., entrent dans cet ensemble lexico-sémantique qui forme autant de sous-branches hyponymiques. On peut considérer que voile, non marqué, est un hyponyme de la composition donnée dans le texte de loi comme les autres «signes religieux» signalés plus haut. Hidjab, d'un emploi marqué (marquage de l'origine arabe de l'emprunt), et foulard (d'une valeur neutre; voir Petiot, 1995) sont cohyponymes; burqa et tchador, étant particulièrement marqués et situés par rapport aux pays concernés (Afghanistan, Iran), ne se situent pas sur le même plan.

Ce trait religieux du voile est clairement rapporté par La Nouvelle République du Centre Ouest (24 février 2005), qui présente une version abrégée de la longue histoire du voile pour conclure au « fondement coranique du voile» :

Qu'on l'appelle voile, foulard, haïk, hidjab, khimar ou encore tchador, son histoire vient du fond des âges. Une histoire bien antérieure à celle du Coran. Dès lors, l'idée selon laquelle «le Coran a obligé les femmes à porter le voile» est selon certains auteurs à inscrire au catalogue des idées fausses [C'est la thèse défendue par Odon Vallet, enseignant à la Sorbonne et à l'université Paris 7, dans son Petit lexique des idées fausses sur les religions]. N'en déplaise aux islamistes qui en ont fait leur bannière, le voile serait apparu vers l'an 1000 avant J.-C. en Assyrie [...] La Bible hébraïque fait d'une tête non voilée un symbole de prostitution. Reste que le premier à imposer cet accessoire aux femmes n'est autre que saint Paul, au ler siècle. L'Épître aux Corinthiens dit que, pour prier, « la femme doit porter sur la tête la marque de sa dépendance».

Au début du $7^{\mathrm{e}}$ siècle vint l'islam. Le Coran consacre au voile deux passages explicites.

15. L’hypo/hyperonymie permet des inclusions sémantiques: "L'hyponymie, comme le prouve la création du terme par analogie [Lyons, 1970, p. 347] avec ceux de synonymie et d'antonymie, a pour objectif premier de cerner une relation sémantique entre des unités lexicales. De quel type de relation sémantique s'agit-il ? Tous les commentateurs rappellent qu'il ne s'agit pas d'une relation nouvelle engendrée par le développement parfois turbulent des théories sémantiques actuelles, mais du rapport bien connu qui unit un lexème comme tulipe, appelé hyponyme, à un lexème comme fleur, appelé hyperonyme ou encore superordonné, un rapport d'inclusion donc et d'inclusion sémantique. »(Kleiber et Tamba, 1990, p. 7-8) 
Dans le premier, on demande aux croyantes de « rabattre leur étoffe sur leur poitrine », dans le second il est recommandé aux épouses et filles du Prophète de « ramener sur elles de grandes étoffes » pour ne pas être importunées par les hommes.

Le voile a donc un fondement coranique. [...]

L'antériorité du voile dans les pays méditerranéens est également attestée par Tite-Live dans le véritable réquisitoire auquel se livre Caton l'Ancien à l'encontre des matrones romaines, sorties sans cacher leur chevelure pour prévenir les Romains de l'attaque du Capitole. Venu de la culture méditerranéenne, le voile s'adapte aux religions et devient « le » signe des femmes.

Dans le corpus contemporain, un nouvel hyperonyme apparait, signes ou tenues par lesquels les élèves manifestent ostensiblement une appartenance religieuse. Il réorganise dès lors l'ensemble des relations sémantiques des vocables rencontrés.

Le mot hidjab peut être présenté comme un xénisme, mais il connait une " acclimatation » linguistique. Le référent original, le vêtement, est peu présent dans l'Hexagone; c'est pourquoi, par glissement métonymique, les discours médiatiques comme les discours lexicographiques ne retiennent, au niveau sémantique, qu'une partie du vêtement, le voile. C'est bien ce que laissent entendre certaines gloses discursives et nombre de reformulations. Hidjab, au moins dans cette acception métonymique, dénomme non pas une réalité inexistante en France mais une réalité nouvelle; l'étiquette de «xénisme» apparait dès lors partiellement inadéquate. De leur côté, les mots voile et foulard ont élargi leur polysémie sous forme de néologie sémantique puisqu'ils peuvent nommer cette nouvelle réalité ; le voile a une nouvelle acception, celle de voile islamique, de même que le foulard « intègre » un nouveau référent, le foulard islamique. Ce phénomène semble présent dans l'exemple suivant : "L'épreuve de force de la République avec les adeptes du "foulard" » (Le Figaro, "Voile et kippa, même combat? », 9 mai 2003). Les guillemets créent une mise à distance du mot; cette modalisation autonymique invite à un retour sur la forme et le sens du mot, ce qui permet au rédacteur et au lecteur de « réajuster » ce qu'il est possible d'entendre aujourd'hui par foulard.

Le port d'un foulard - prenant une nouvelle forme dans notre société - par certaines adolescentes dans le cadre de l'école a entraîné des actions politiques et des débats qui se sont notamment manifestés dans les discours de presse. Nous avons pu voir que la nomination de ce foulard ne va pas de soi : plusieurs noms entrent en concurrence et le choix de telle ou telle dénomination positionne le locuteur dans le débat. Si la glose a pu accompagner le mot hidjab 
pour présenter une définition - assez rarement - ou pour donner un équivalent - comme foulard (islamique) ou voile (islamique) -, son emploi n'est pas systématique ${ }^{16}$. L'apparition du mot hidjab dans les discours puis dans le lexique français invite à "repositionner » la valeur sémantique des mots entrant dans le champ des coiffures, mais aussi dans celui des signes religieux. Au-delà de ces phénomènes vient s'ajouter, dans un autre espace discursif que celui de la presse, un texte de loi. Il y a une mise en ordre. Par ailleurs, en proposant un regroupement qui n'avait pas encore été pensé et avec l'émergence d'une « étiquette » générique, celle des « signes ou tenues manifestant une appartenance religieuse ", l'Institution construit ici une nouvelle conception et une nouvelle composition linguistique. Ce passage par le législatif, qui règle des situations en s'appuyant sur des formules linguistiques, semble constituer ce que J. Guilhaumou ${ }^{17}$ appelle un "évènement linguistique ». Ce texte a un impact : il doit mettre un terme au débat et il participe à une nouvelle organisation lexicosémantique.

\section{Références}

AUTHIER-REVUZ J., 2003, "Le fait autonymique: langage, langue, discours. Quelques repères », Parler des mots, Paris, Sorbonne nouvelle, p. 67-96.

— DOURY M. et REBOUL-TOURÉ S. éd., 2003, Parler des mots. Le fait autonymique en discours, Paris, Sorbonne nouvelle.

BOYER H., 1993, "Tchador, les mots de "l'affaire” », Médiascopie, n 4 , p. 67-72.

CHARAUDEAU M. et MAINGUENEAU D. éd., 2002, Dictionnaire d'analyse du discours, Paris, Le Seuil.

DETRIE C., SIBLOT P. et VERINE B. éd., 2001, Termes et concepts pourl'analyse du discours. Une approche praxématique, Paris, Champion.

DOURY M., 2003, "Argumentation et mise en voix; les discours quotidiens sur l'immigration », Marina Bondi et Sorin Stati éd., Dialogue Analysis 2000, Selected Papers from the 1oth IADA Anniversary Conference, Bologna 2000, Tübingen, Niemeyer, p. $173-183$.

— 2004, "La fonction argumentative des échanges rapportés », Lopez-Munoz J.-M., Marnette S. et Rosier L. éd., Le discours rapporté dans tous ses états, Paris, L'Harmattan.

KERBRAT-ORECCHIONIC., 1980, "La polémique et ses définitions ", Le discours polémique, Lyon, Presses universitaires de Lyon, p. 3-40.

KLEIBER G., TAMBA I., 1990, «L'hyponymie revisitée : inclusion et hiérarchie », Langages, $\mathrm{n}^{\circ}$ 98, Paris, Larousse, p. 7-32.

LYONS J., 1970, Linguistique générale, Paris, Larousse.

16. Nous n'avons pas noté, sur la courte diachronie étudiée, un emploi massif de la glose au début de l'apparition du mot hidjab et qui se serait ensuite effacé dès le mot connu.

17. Notamment dans Charaudeau et Maingueneau, 2002, p. 247-249. 
MORTUREUX M.-F., 1999, La lexicologie entre langue et discours, Paris, Sedes.

-2001, «Xénisme», Une langue: le français, Tomassone R. éd., Paris, Hachette Éducation.

MARTIN R., 1990, "La définition “naturelle” », La définition, Paris, Larousse, p. 86-95.

PETIOTG., 1995, "Voile, tchador ou foulard? Problèmes de dénomination dans des discours médiatiques ", Les Carnets du Cediscor, $\mathrm{n}^{\circ}$ 3, p. 43-62.

SIBLOT P., 1992, «Ah ! qu'en termes voilés ces choses-là sont mises », Mots. Les langages du politique, $\mathrm{n}^{\circ} 30, \mathrm{p} .5-16$.

STEUCKARDT A., 2003, "Présentation », Steuckardt et Niklas-Salminen éd. (voir suiv.), p. 5-17.

— et NIKLAS-SALMINEN A. éd., 2003, Le mot et sa glose, Aix-en-Provence, Publications de l'Université de Provence.

— et NIKLAS-SALMINEN A. éd., 2005, Les marqueurs de glose, Aix-en-Provence, Publications de l'Université de Provence.

REY A., 1979, La terminologie, noms et notions, Paris, PUF, «Que sais-je ». 


\section{Annexe 1}

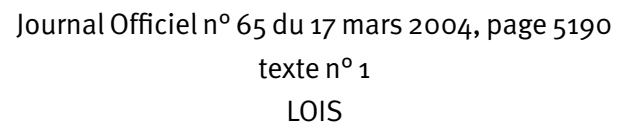

LOI n $2004-228$ du 15 mars 2004 encadrant, en application du principe de laïcité, le port de signes ou de tenues manifestant une appartenance religieuse dans les écoles,

collèges et lycées publics (1)

NOR: MENX0400001L

L'Assemblée nationale et le Sénat ont adopté, Le Président de la République promulgue la loi dont la teneur suit :

Article 1

Il est inséré, dans le code de l'éducation, après l'article L. 141-5, un article L. 141-5-1 ainsi rédigé :

«Art. L. 141-5-1. - Dans les écoles, les collèges et les lycées publics, le port de signes ou tenues par lesquels les élèves manifestent ostensiblement une appartenance religieuse est interdit.

Le règlement intérieur rappelle que la mise en oeuvre d'une procédure disciplinaire est précédée d'un dialogue avec l'élève. »

(http://www.legifrance.gouv.fr/html/actualite/actualite_legislative/2004-228/laicite. htm)

\section{Annexe 2}

Titres des articles parus dans la presse française en 2005 (à partir de la base Factiva)

Alternatives Economiques

$1^{\text {er }}$ mars : « Le multiculturalisme, une réalité des sociétés modernes »

LaCroix

8 novembre: « Mona et Makarem, deux Égyptiennes en campagne. L'une est chrétienne, l'autre islamiste. Mona Makram-Ebeid et Makarem Al Deiri se présentent aux législatives égyptiennes dont le premier tour a lieu demain. » Le Caire, reportage de notre envoyée spéciale. 
29 novembre: «Dossier. Les banlieues des autres. Allemagne. À Berlin, “chaque communauté vit de son côté”. Il y a tout juste un mois éclatait la révolte dans les banlieues françaises. La Croix est allée voir chez différents voisins européens - notamment à Berlin - à quelles difficultés ils étaient confrontés. » En France, où l'enseignement a été mis en accusation, le gouvernement ouvre cette semaine trois voies de réforme. Berlin, reportage de notre envoyé spécial.

\section{Les Échos}

5 septembre : «Égypte : le redoux politique»

\section{L'Express}

22 septembre: «Afghanistan. La loi des tribus»

6 octobre: «Proche-Orient. Hadassah ou la paix sous perfusion»

20 octobre : «Égypte. Les tourments du “Pharaon” »

\section{Le Figaro}

26 janvier : «Le Hamas tente de s'allier avec la femme de Barghouti »

8 février : «Les paradoxes du voile iranien»

19 février : «L'humanisme laïque »

17 juin : «Des étudiantes voilées créent des incidents »

8 juillet : "L'antiterrorisme français sur le pied de guerre »

19 septembre : «Au lycée Averroès, les résultats au bac sont jugés “corrects” »

19 novembre : « Le Canada tire sa prospérité de l’immigration »

\section{Libération}

17 novembre : «Une carte de séjour refusée pour cause de voile »

18 novembre : «Polygamie française »

\section{Le Monde}

25 juin : "En rentrant au pays, des expatriés se découvrent une âme de "pionnier” " 26 juin: «Le GSPC algérien menacerait la France dans le cadre du "djihad" international»

12 aout: « Mesdames les imams»

27 septembre : «Une “nuit contre l'oubli” des disparus d’Algérie»

29 septembre: «En Algérie, dans la Mitidja, ni pardon ni oubli »

29 novembre : «Ya-t-il un néolibéralisme islamique? »

\section{Le Nouvel Observateur}

10 mars : «Mahomet et les femmes »

27 octobre : «Trois mois après les attentats de Londres... Islam : Le défi de Tony Blair »

\section{Le Parisien}


6 décembre : «Bobigny. La maman voilée indésirable à l'école » 17 décembre : «Bondy. Les cours privés d'arabe font le plein » Le Point

3 mars : "La révolution du Cèdre»

6 octobre : "La fièvre capitaliste »

3 novembre: «Sus à l'“obscurantisme de gauche” ! ”

15 décembre : «Ce voile qui dérange» 